\title{
NAKAYAMA ALGEBRAS AND GRADED TREES
}

\author{
BY
}

B. ROHNES AND S. O. SMAL $\varnothing$

\begin{abstract}
Let $k$ be an algebraically closed field. We show that if $T$ is a finite tree, then there is a grading $g$ on $T$ such that $(T, g)$ is a representation finite graded tree, and such that the corresponding simply connected $k$-algebra is a Nakayama algebra (i.e. generalized uniserial algebra).
\end{abstract}

Introduction. Let $k$ be an algebraically closed field. A simply connected algebra $\Lambda$ over $k$ is an algebra which is representation-finite, connected, basic, finite-dimensional and has a simply connected Auslander-Reiten quiver $\Gamma_{\Lambda}$. In order to study the simply connected algebras, K. Bongartz and P. Gabriel introduced the notion of graded trees [2]. If $T$ is a finite tree, let $T_{0}$ denote the set of vertices of $T$. A grading of the tree $T$ is a function $g: T_{0} \rightarrow N$ ( $N$ is the nonnegative integers), satisfying the following conditions:

(a) $g(x)-g(y) \in 1+2 Z$, whenever $x$ and $y$ are neighbours in $T$ ( $Z$ the integers).

(b) $g^{-1}(0) \neq \varnothing$.

A graded tree is a pair $(T, g)$ formed by a tree $T$ and a grading $g$ of $T$.

K. Bongartz and P. Gabriel show that there is a bijection between the isomorphism classes of representation-finite graded trees and the isomorphism classes of simply connected algebras. For the benefit of the reader we give a summary of their results in $\S 1$. They also show in [2] that every tree $T$ admits only a finite number of representation-finite gradings. In this paper we show that for every tree $T$ it is possible to find a grading $g$ such that $(T, g)$ is representation-finite. This answers a question raised by $P$. Gabriel. In fact, what we show is that given a tree $T$ it is possible to find a grading $g$ such that the associated simply connected algebra is a Nakayama algebra. Conversely, given a noncyclic Kupisch series for a Nakayama $k$-algebra $\Lambda$, one may associate a graded tree $(T, g)$ such that the simply connected $k$-algebra obtained from $(T, g)$ is $\Lambda$.

1. Simply connected algebras and graded trees. Let $(T, g)$ be a graded tree. To this graded tree we associate a translation quiver $Q_{T}$ in the following way. The vertices of $Q_{T}$ are the points $(n, t) \in N \times T_{0}$ such that $n-g(t) \in 2 N$, two such vertices $(m, s)$ and $(n, t)$ are joined by an arrow $(m, s) \rightarrow(n, t)$ if $s, t$ are neighbours in $T$ and $n=m+1$. The projective vertices are the points $(g(t), t)$, the translate of a nonprojective vertex is defined by $\tau(n, t)=(n-2, t)$.

Received by the editors September 13, 1982.

1980 Mathematics Subject Classification. Primary 16A64, 16A46.

Key words and phrases. Simply connected algebra, module, graded tree, Kupisch series. 
For each graded tree $T=(T, g)$ there is a unique map $d:\left(Q_{T}\right)_{0} \rightarrow N^{T_{0}}$ satisfying the following conditions:

(a) $d(g(t), t)=\delta_{t}+\Sigma_{s} d(g(t)-1, s)$, where $s$ ranges over the neighbours $s$ of $t$ such that $g(s)<g(t)$ and $d(g(t)-1, s)>0$ (where a function is $>0$ if all its values are $\geqslant 0$ and at least one of them if $>0$ ), and the Kronecker function $\delta_{t}$ takes the value 1 at $t$ and 0 otherwise.

(b) $d(n, t)=\Sigma_{s} d(n-1, s)-d(n-2, t)$, whenever $(n, t)$ is a nonprojective vertex of $Q_{T}$ for which the functions $d(n-2, t)$ and $\Sigma_{s} d(n-1, s)-d(n-2, t)$ are both $>0$, when $s$ ranges over the neighbours of $t$ in $T$ such that $g(s)<n$.

(c) For any other vertex $(n, t)$ of $Q_{T}$ we have $d(n, t)=0$.

Using these conditions, $d(n, t)$ can be computed by induction on $n$, starting with $n=g(t) . d$ is called the dimension map of $Q_{T}$. We denote by $R_{T}$ the full subtranslation-quiver of $Q_{T}$ formed by the vertices $(n, t)$ such that $d(n, t)>0$. The grading $g$ is called admissible if $R_{T}$ is a connected subquiver of $Q_{T}$, and $T$ is then called an admissible graded tree. The grading is called representation-finite if it is admissible and $R_{T}$ is finite. $T$ is then called a representation-finite graded tree.

REMARK. We are using a definition of $d$ different from the one given in [2, p. 356], since it was through our definition we saw the main result of this paper. Also with our definition the projective vertices in $R_{T}$ coincide with those in $Q_{T}$ regardless of the grading $g$. It is easy to see that the two definitions are the same when $R_{T}$ is connected.

Let $T$ be an admissible graded tree. Let $A^{T}$ be the finite-dimensional algebra $A^{T}=\amalg_{p, q} k\left(R_{T}\right)(q, p)$, where $k\left(R_{T}\right)$ is the mesh category of $R_{T}$, and $p, q$ range over all projective vertices of $R_{T}$. Then each vertex $x$ of $R_{T}$ is associated with an $A^{T}$-module $M(x)=\amalg_{p} k\left(R_{T}\right)(p, x)$, where $p$ ranges over all projective vertices of $R_{T}$, and it is shown in [2] that for every vertex $(n, t)$ of $R_{T}$, the $A^{T}$-module $M(n, t)$ is indecomposable and its dimension vector is $d(n, t)$, especially, $M(g(t), t)$ are the indecomposable projective modules, and if $M(n, t)$ is not projective, $\operatorname{DTr}(M(n, t))$ $=M(n-2, t)=M(\tau(n, t))$. In fact, if $(T, g)$ is representation-finite, then there is a translation-quiver isomorphism of the Auslander-Reiten quiver $\Gamma_{A^{T}}$ onto $R_{T}$.

If $\Gamma$ is a locally finite translation-quiver, and $x$ is a vertex of $\Gamma$, the set of all $n \in Z$ such that $\tau^{n} x$ is defined, is an inverval $\mathscr{D}$ of $Z$. Then the set $x^{\tau}=\left\{\tau^{n} x, n \in \mathscr{D}\right\}$ is called the $\tau$-orbit of $x$. The vertex $x$ is stable if $\mathscr{D}=Z$, it is periodic if it is stable and has a finite $\tau$-orbit. The $\tau$-orbits of a connected component $E$ of the stable part $\Gamma_{s}$ of $\Gamma$ are either all finite or all infinite. In the first case we call $E$ a periodic component of $\Gamma$.

If $\underset{\alpha}{\rightarrow} y$ is an arrow of $\Gamma$, where $y$ is not projective, there is a unique arrow $\tau y \rightarrow x$, which we denote $\sigma \alpha$. The $\tau$-orbit of $\alpha$, denoted $\alpha^{\sigma}$, is the set of all arrows of $\Gamma$ of the form $\sigma^{m} \alpha$.

The graph $G_{\Gamma}$ associated with $\Gamma$ has as vertices the nonperiodic $\tau$-orbits and the periodic components of $\Gamma$. To each periodic component, considered as a vertex of $G_{\Gamma}$, we associate a loop of $G_{\Gamma}$. Let $\alpha^{\sigma}$ be a $\sigma$-orbit connecting $x^{\tau}$ and $y^{\tau}$. If both $x$ and $y$ are nonperiodic, we associate with $\alpha^{\sigma}$ an edge connecting the vertices $x^{\tau}$ and $y^{\tau}$. If $y$ is not periodic and $x$ belongs to a periodic component $E$ we associate with $\alpha^{\sigma}$ an edge of $G_{\Gamma}$ connecting $E$ and $y^{\tau}$. 
Now, if $A$ is a simply connected algebra, and $\Gamma_{A}$ is the Auslander-Reiten quiver of $A$, then the graph $G_{A}$ associated with $\Gamma_{A}$ is a tree [2, Theorem 4.2]. Since $\Gamma_{A}$ is simply connected and finite, there is a unique quiver morphism $K_{A}: \Gamma_{A} \rightarrow Z A_{2}$ such that $0=\operatorname{Min} K(x)$, the minimum taken over all vertices $x$ of $\Gamma_{A}$. Here $Z A_{2}$ is the following translation quiver where $-\rightarrow$ indicates the translation. Since $G_{A}$ is a tree, each $\tau$-orbit $t$ of $\Gamma_{A}$ contains exactly one projective vertex $p_{t}$. We set $g_{A}(t)=$ $K_{A}\left(p_{t}\right) \in N$. The function $g_{A}$ is then a grading of $G_{A}$, and $\left(G_{A}, g_{A}\right)$ is a graded tree. The maps $(T, g) \rightarrow A^{T}$ and $A \rightarrow\left(G_{A}, g_{A}\right)$ are inverse maps and therefore there is a bijection between the isomorphism classes of representation-finite graded trees and the isomorphism classes of simply connected algebras $[2,6.5]$.

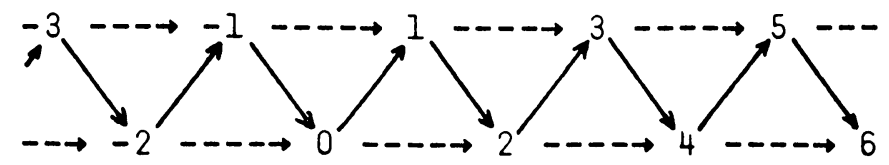

$Z A_{2}$

2. The relation between Kupisch series and trees. In this section we examine the relation between the Nakayama algebras with noncyclic Kupisch series and trees. We show that given a tree $T$, it is possible to associate a Nakayama algebra $\Lambda$ to this tree such that the graph of $\Lambda$ is isomorphic to $T$. From this follows the main result of this paper: To every tree $T$ it is possible to find a grading $g$ such that $(T, g)$ is representation-finite. The Nakayama algebra $\Lambda$ is not uniquely given by the construction we use.

But first we show how to construct a tree $T_{\Lambda}$ from a Nakayama algebra $\Lambda$ with noncyclic Kupisch series. The construction determines $T_{\Lambda}$ uniquely up to isomorphism, and later we will see that $T_{\Lambda}$ is in fact the graph $G_{\Lambda}$ associated with $\Gamma_{\Lambda}$. Therefore this gives us an easy way to construct $G_{\Lambda}$ if $\Lambda$ is a Nakayama algebra.

We recall that the Kupisch series for an indecomposable Nakayama algebra $\Lambda$ is an ordered complete set of representatives $P_{1}, \ldots, P_{n}$ of the isomorphism classes of indecomposable projective $\Lambda$-modules, satisfying the following conditions:

(i) $P_{i} / \underline{r} P_{i} \cong r P_{i+1} / \underline{r}^{2} P_{i+1}$, or equivalently:

$$
P_{i+1} / \underline{r} P_{i+1}=\operatorname{Tr} D\left(P_{i} / \underline{r} P_{i}\right) \text {. }
$$

(ii) $L\left(P_{i}\right) \geqslant 2$ for all $i$ such that $2 \leqslant i \leqslant n$.

(iii) $L\left(P_{i+1}\right) \leqslant L\left(P_{i}\right)+1$ for $i=1, \ldots, n$, and $L\left(P_{1}\right) \leqslant L\left(P_{n}\right)+1$.

( $L(M)=$ the length of the $\Lambda$-module $M$.)

Any finite sequence of integers $c_{1}, \ldots, c_{n}$ satisfying (ii) and (iii) above when we put $c_{i}=L\left(P_{i}\right)$, is called an admissible sequence. Given an arbitrary admissible sequence, an algebra can be constructed such that its Kupisch series corresponds to this sequence. The Kupisch series is noncyclic if $L\left(P_{1}\right)=1$. For details, see [4].

Let $\Lambda$ be an indecomposable Nakayama algebra with a noncyclic Kupisch series. Let $T_{\Lambda}$ be the following tree: The vertices of $T_{\Lambda}$ are the representatives of the isomorphism classes of indecomposable projective $\Lambda$-modules. For each $i$, let $t_{i}$ be the vertex corresponding to the projective $P_{i}$. If $i, j \in\{1, \ldots, n\}$, with $i \leqslant j$, there is an edge connecting $t_{i}$ and $t_{j}$ if $i$ is the greatest integer less than $j$ such that $L\left(P_{j}\right)=L\left(P_{i}\right)+1 . T_{\Lambda}$ is connected, since for every $j \in\{2, \ldots, n\}$ it follows from 
(iii) above that there always exists such an $i$, and it is not difficult to see that $T_{\Lambda}$ really is a tree when constructed as above.

We define a walk in a tree $T$ to be a sequence of vertices $S_{1} \cdots S_{n}$, connected by edges $\alpha_{1} \cdots \alpha_{n-1}$ in such a way that for each $i \in\{1, \ldots, n-1\}, S_{i}$ and $S_{i+1}$ are connected by the edge $\alpha_{i}$ of $T$.

If $u$ is a walk: $S_{1}-S_{2}-\cdots \stackrel{\alpha_{k-1}}{-} S_{k}$ in a tree $T$, we define the length of $u$, $l(u)=k$. If $S_{i}$ and $S_{j}$ are two vertices of $T$, the shortest walk from $S_{i}$ to $S_{j}$ is the walk that does not pass through any vertex twice. It follows from the construction above that for any vertex $t_{i}$ in $T_{\Lambda}, L\left(P_{i}\right)$ is equal to the length of the shortest walk in $T_{\Lambda}$ from $t_{i}$ to $t_{1}$.

EXAMPLE. Given the admissible sequence $\{1,2,3,3,4,3\}$ the corresponding tree $T_{\Lambda}$ is:

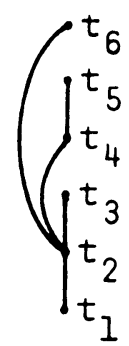

Conversely, starting with a tree $T$, to this tree we can associate a noncyclic Kupisch-series for an indecomposable Nakayama algebra: Fix a point $t_{1}$ in the tree $T$ and a walk $V$ around the tree from $t_{1}$ to $t_{1}$ which passes through every edge in the tree exactly twice.

EXAMPLE. If $T$ is the tree, then $V: t_{1}-B-A-B-C-D-C-E-C-B-t_{1}-F-t_{1}-G-H-G-$ $t_{1}-I-t_{1}$ is such a walk.

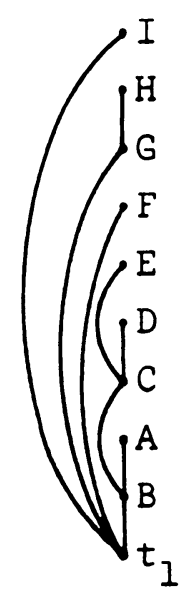

The order in which $V$ passes through each vertex for the first time defines an ordering of the vertices of $T$, such that $t_{i}$ is the $i$ th new vertex which occurs in $V$. In 
the example above $t_{2}=B, t_{3}=A, t_{4}=C, t_{5}=D, t_{6}=E, t_{7}=F, t_{8}=G, t_{9}=H$, $t_{10}=I$.

Suppose $T_{0}=\left\{t_{1}, \ldots, t_{n}\right\}$. Then for each $i \in\{1, \ldots, n\}$, let $C_{i}=l\left(U_{i}\right)$, where $U_{i}$ is the shortest walk in $T$ from $t_{1}$ to $t_{i}$. It is clear that $C_{1}=1$, and that $C_{i} \geqslant 2$ for $i \geqslant 2$. Further, if $t_{i+1}$ is a neighbour of $t_{i}$, then $C_{i+1}=C_{i}+1$, because it is clear that there is only one neighbour $t_{k}$ of $t_{i}$ with $l\left(U_{k}\right)<l\left(U_{i}\right)$, and it is the only neighbour with $k<i$. If $t_{i+1}$ is not a neighbour of $t_{i}$, then $t_{i+1}$ is a neighbour of a vertex $t_{j}$ with $l\left(U_{j}\right)<l\left(U_{i}\right)$. So in that case $C_{i+1}<C_{i}+1$. Therefore we have that $\left\{C_{1}, \ldots, C_{n}\right\}$ is an admissible sequence which corresponds to the noncyclic Kupisch series of an indecomposable Nakayama algebra.

We now claim that every indecomposable Nakayama algebra $\Lambda$ with a noncyclic Kupisch series is simply connected. The ordinary quiver $Q_{\Lambda}$ of an indecomposable Nakayama algebra $\Lambda$ with a noncyclic Kupisch series is a tree of form $\rightarrow \cdots \rightarrow$, therefore the fundamental group $\pi\left(Q_{\Lambda}, x\right)=\{1\}$, and from [3,2.2] we know that there is a surjective group homomorphism $\phi_{x}: \pi\left(Q_{\Lambda}, x\right) \rightarrow \pi\left(\Gamma_{\Lambda}, x\right)$. Therefore $\pi\left(\Gamma_{\Lambda}, x\right)$ is trivial, and $\Lambda$ is simply connected. See also $[2,6.1]$.

Therefore, to every tree $T$ one may associate a simply connected algebra $\Lambda$, namely, the indecomposable Nakayama algebra constructed above. Remark that the Kupisch series of $\Lambda$ depends on the choice of the basis point $t_{1}$ and the walk $V$, therefore given a tree $T$, there is usually more than one choice of a corresponding Nakayama algebra $\Lambda$. For our purposes, it is enough to look at one of these. Since $\Lambda$ is simply connected, we know that the graph $G_{\Lambda}$ is a tree [2, Theorem 4.2]. Because of the connection between simply connected algebras and graded trees, to show that the tree $T$ has a representation-finite grading, it is enough to show that $G_{\Lambda}$ is isomorphic to the tree $T$. (Remark that we consider a tree to be completely determined by the vertices and the edges connecting them, such that for instance, are considered to be isomorphic.)
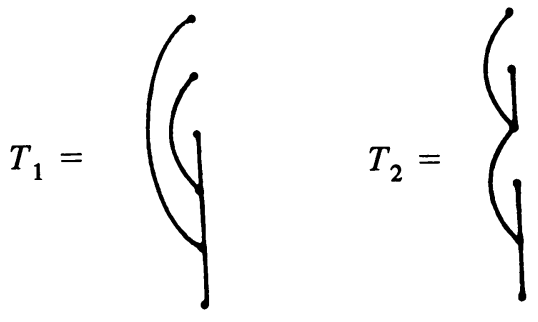

The number of $\tau$-orbits is equal to the number of projective $\Lambda$-modules, so the number of vertices of $G_{\Lambda}$ is equal to the number of vertices of $T$. Now we define a map $\theta: T_{0} \rightarrow\left(G_{\Lambda}\right)_{0}$ such that $\theta\left(T_{i}\right)$ is the vertex representing the $\tau$-orbit of the projective $\Lambda$-module $P_{i}$ with $L\left(P_{i}\right)=C_{i}$, where $C_{i}$ is as defined above. Then $\theta$ is a bijection. Denote $\theta\left(t_{i}\right)$ by $S_{i}$. Since $G_{\Lambda}$ and $T$ are trees with the same number of vertices, they also have the same number of edges, and to prove that $G_{\Lambda}$ is isomorphic to $T$, it is enough to show that if there is an edge connecting the vertices $t_{i}$ and $t_{j}$ in $T$, there is an edge connecting the vertices $S_{i}$ and $S_{j}$ in $G_{\Lambda}$. 
Let us recall some useful facts about Nakayama algebras. If $\Lambda$ is a Nakayama algebra, then every indecomposable $\Lambda$-module is of the form $P_{i} / \underline{r}^{k} P_{i}$, where $k \geqslant 0$ and $P_{i}$ is an indecomposable projective $\Lambda$-module. If $P_{i} / \underline{r}^{k} P_{i}$ is an indecomposable nonprojective $\Lambda$-module, then it is shown in [1] that the almost split sequence with $P_{i} / r^{k} P_{i}$ as right-hand term has the form

$$
0 \rightarrow P_{i-1} / \underline{r}^{k} P_{i-1} \rightarrow P_{i-1} / \underline{r}^{k-1} P_{i-1} \amalg P_{i} / \underline{r}^{k+1} P_{i} \rightarrow P_{i} / \underline{r}^{k} P_{i} \rightarrow 0 .
$$

It follows from this that $\tau$-orbits preserve the length of modules, and all simples belong to the same $\tau$-orbit. We also recall that given the Kupisch series for a Nakayama algebra $\Lambda$, we always have an epimorphism $P_{i} \rightarrow \underline{r} P_{i+1}$. If $L\left(P_{i+1}\right)=$ $L\left(P_{i}\right)+1$, this epimorphism is also an isomorphism.

Now, suppose that $t_{i}$ or $t_{j}$ is $t_{1}$, say $t_{i}=t_{1} . L\left(P_{1}\right)=1$, so $P_{1}$ is the unique simple projective. Since $t_{j}$ is a neighbour of $t_{1}$, we see from the construction above, that $L\left(P_{j}\right)=2$. But that means $\underline{r} P_{j}$ is simple, and then either $r P_{j} \cong P_{1}$, or $\underline{r} P_{j}$ is in the $\tau$-orbit determined by $P_{1}$, so $S_{j}$ is a neighbour of $S_{1}$ in $G_{\Lambda}$. Suppose that neither $t_{i}$ nor $t_{j}$ is $t_{1}$, but that there is an edge $t_{i}-t_{j}$. Let $i<j$. Then $L\left(P_{j}\right)=L\left(P_{i}\right)+1$ by the construction above. Therefore $L\left(\underline{r} P_{j}\right)=L\left(P_{i}\right)$. Since $\Lambda$ is Nakayama, $\underline{r} P_{j}$ belongs to the $\tau$-orbit of a projective module with the same length as $P_{i}$. We remember that the ordering of the projectives was defined by help of the walk $V$ in $T$, and since $T$ is a tree, and every edge in $T$ appears in $V$ exactly twice, we have $L\left(P_{k}\right)>L\left(P_{i}\right)$ for every edge $k$ such that $i<k<j$. If $P_{m}$ is an indecomposable $\Lambda$-module, the length of the $\tau$-orbit determined by $P_{m}, l\left(P_{m}^{\tau}\right)$, is the number of nonisomorphic objects in the $\tau$-orbit. For a Nakayama algebra $\Lambda$, the following formula is easily obtained, using the form of almost split sequences indicated above: $l\left(P_{m}^{\tau}\right)=h-m+1$, where $h$ is maximal with the property that $L\left(P_{p}\right)-L\left(P_{m}\right)>0$ for all $p$ such that $m<p \leqslant h$. Further if $L\left(P_{m}\right)=q$, then the modules in this $\tau$-orbit are the modules of the form $P_{p} / \underline{r}^{q} P_{p}$, where $m \leqslant p \leqslant h$. In our case, if we let $L\left(P_{i}\right)=q$, it follows that $P_{j-1} / \underline{r}^{q} P_{j-1}$ is in the $\tau$-orbit of $P_{i}$. But since we have an epimorphism $P_{j-1} \rightarrow \underline{r} P_{j}$, and $L\left(\underline{r} P_{j}\right)=L\left(P_{i}\right)=q$, we have $\underline{r} P_{j} \cong P_{j-1} / \underline{r}^{q} P_{j-1}$. Therefore $\underline{r} P_{j}$ is in the $\tau$-orbit of $P_{i}$, and we have an edge $S_{i}-S_{j}$ in $G_{\Lambda}$.

We have now proved the main result of this paper:

THEOREM. If $T$ is a finite tree, then there is a grading $g$ such that $(T, g)$ is representation-finite, and such that the corresponding simply connected algebra $\Lambda$ is a Nakayama algebra.

EXAMPLE. Let $T$ be the tree:

$$
\int\left(\begin{array}{l}
t_{8} \\
\int_{7}^{t_{6}} \\
t_{5} \\
t_{4} \\
t_{3}^{t_{2}} \\
t_{1}
\end{array}\right.
$$


Let $V$ be the walk: $t_{1}-t_{2}-t_{1}-t_{3}-t_{1}-t_{4}-t_{5}-t_{6}-t_{5}-t_{5}-t_{7}-t_{4}-t_{8}-t_{4}-t_{1}$, which is a walk around the tree, passing through every edge in the tree exactly twice. To the tree $T$ and the walk $V$ we may associate the Kupisch series $\left\{P_{1}, P_{2}, P_{3}, P_{4}, P_{5}, P_{6}, P_{7}, P_{8}\right\}$ corresponding to the admissible sequence $\{1,2,2,2,3,4,3,3\}$.

The $A R$-quiver $\Gamma_{\Lambda}$ of the Nakayama algebra $\Lambda$ is the following:

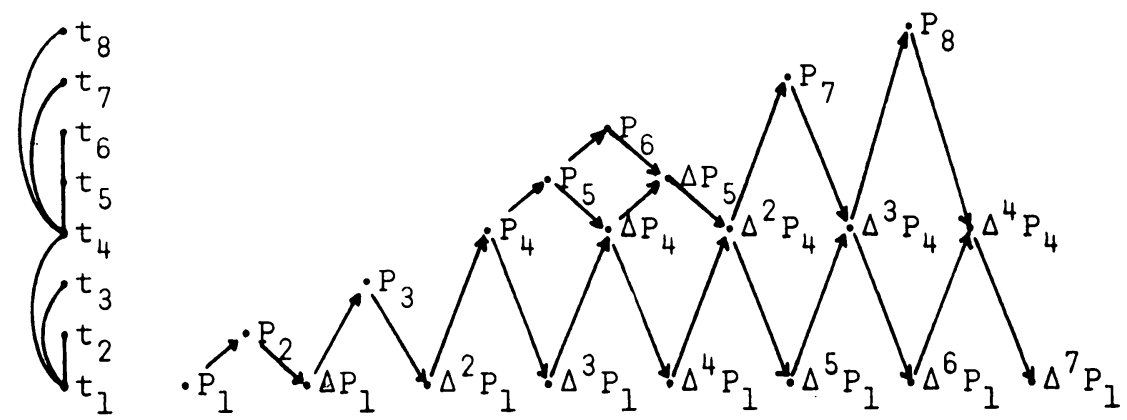

We see that $P_{2}, P_{3}, P_{6}, P_{7}$ and $P_{8}$ all are projective injectives. All arrows pointing upward correspond to irreducible monomorphisms, all arrows pointing downward correspond to irreducible epimorphisms. If there is an irreducible monomorphism $X \rightarrow Y, L Y=L X+1$, and if there is an irreducible epimorphism $X \rightarrow Y, L X=L Y$ +1 . If $X \in$ ind $\Lambda$, Soc $X$ is the unique simple module $S$ such that there is a chain of irreducible monomorphisms $S \rightarrow \cdots \rightarrow X, X / \underline{r} X$ is the simple module $T$ such that there is a chain of irreducible epimorphisms $X \rightarrow \cdots \rightarrow T . L(X)$, the length of $X$, is equal to the shortest walk in $\Gamma_{\Lambda}$ from Soc $X$ to $X$.

If we start with a tree $T$, choose a point $t$, and a walk $V$ around the tree, and construct the corresponding Nakayama algebra $\Lambda$ in the way described above, it is possible to find the number of nonisomorphic indecomposable projective injective $\Lambda$-modules just by looking at the tree $T$.

Proposition. The number of projective injective $\Lambda$-modules is equal to the number of vertices in $T$, different from $t_{1}$, which have only one neighbour.

Proof. $P_{i}$ is projective injective if and only if $L\left(P_{i+1}\right)<L\left(P_{i}\right)+1$. If $t_{i} \neq t_{1}$ is a point in $T$ having only one neighbour $t_{j}$, then every walk in $T$ from $t_{1}$ to $t_{i}$ must pass through $t_{j}$, therefore $j<i$, and $t_{i+1}$ is not a neighbour of $t_{i}$. But then $t_{i+1}$ is a neighbour of a point $t_{k}$ which does not lie farther away from $t_{1}$ than $t_{j}$, and $L\left(P_{i+1}\right) \leqslant L\left(P_{j}\right)+1=L\left(P_{i}\right)<L\left(P_{i}\right)+1$, which means that $P_{i}$ is a projective injective module. On the other hand, if $P_{i}$ is a projective injective module, then $L\left(P_{i+1}\right)<L\left(P_{i}\right)+1$, and $t_{i+1}$ is not a neighbour of $t_{i}$. But then $t_{i}$ can have only one neighbour (recall that the walk $V$ that defines the ordering passes through every edge exactly twice). The relation between $V$ and $\Gamma_{\Lambda}$ can be described in the following manner.

Proposition. Let $\theta$ be a chain of irreducible maps in $k\left(\Gamma_{\Lambda}\right)$ given by

$$
\begin{aligned}
\theta: P_{1}=M\left(0, t_{1}\right) & \rightarrow P_{2} \rightarrow \cdots \rightarrow P_{i} \rightarrow \cdots \rightarrow r P_{i+1} \rightarrow \cdots \\
\rightarrow P_{n} & \rightarrow \cdots \rightarrow M\left(2(n-1), t_{1}\right)
\end{aligned}
$$


which passes through all the projectives in the order given by the Kupisch series, and which satisfies the condition that if $P_{n}$ is the last projective in the ordering, then $P_{n} \rightarrow \cdots \rightarrow M\left(2(n-1), t_{1}\right)$ is the unique path from the projective injective module $P_{n}$ to the simple injective $\Lambda$-module $M\left(2(n-1), t_{1}\right)$. Then $V$ is the walk in $T$ constructed by taking for each module in $\theta$ the corresponding point in $T$, and passing through the points in the order defined by $\theta$.

Proof. This can be proven in the same way as the main theorem above.

\section{REFERENCES}

1. M. Auslander and I. Reiten, Representation theory of artin algebras. IV; Invariants given by almost split sequences, Comm. Algebra 5 (1977), 443-518.

2. K. Bongartz and P. Gabriel, Covering spaces in the representation theory, Invent. Math. 65 (1982), $331-378$.

3. P. Gabriel, The universal cover of a representation-finite algebra, Proc. Third Internat. Conf. Rep. Algebra, Puebla, 1980.

4. H. Kupisch, Beiträge zur Theorie nichthalbeinfacher Ringe mit Minimalbedingung, J. Reine Angew. Math. 201 (1959), 100-112.

Department of Mathematics and Statistics, University of Trondheim, NLHT, 7055 Dragvoll, NoRWAY (Current address of S. O. Smalø)

Current address (B. Rohnes): Department of Mathematics, Brandeis University, Waltham, Massachusetts 02154 